Reprod. Nutr. Dévelop., 1986, 26 (4), 943-967.

\title{
Variabilité de la répartition du tissu musculaire du membre postérieur de l'agneau. - I. Muscles individuels
}

\author{
M. R. ANOUS ( $\left.{ }^{1}\right)$
}

Laboratoire de Recherches sur la Viande, I.N.R.A. 78350 Jouy-en-Josas, France.

\begin{abstract}
Summary. Variability of muscular tissue distribution in the hindlimb of lambs. I. Individual muscles.

Using multivariate analysis of the centered data, this paper describes the extent of variation of the weight distribution of individual muscles in the hindlimb of lambs of $18.9 \pm 3.1 \mathrm{~kg}$ carcass weight. We studied a sample of 52 ram lambs and 36 ewe lambs of different morphological types slaughtered at a comparable stage of body development (same relative state of carcass fattening).

Variability in muscle weight was similar in the two sexes (average $\mathrm{CV}=21.95 \%$ in ram lambs and $22.5 \%$ in ewe lambs) and changed from 14.84 to $43.91 \%$ in the former and from 14.91 to $41.93 \%$ in the latter. Some of the mass variability of the different muscles was related to the total muscle weight of the hindlimb, but a part was also due to intrinsic variation related to the nature of the muscles.

The most discriminant variables of the population were of very low weight : Soleus (SO), Extensor digitorum lateralis (ED), Gemelli (GE) and Quadratus femoris (QF); moderately low weight : Sartorius (SA); or average weight : Vastus medialis (VM). Their order of importance was not the same in the two sexes. The breed groups could be distinguished by the weight ratio values of these muscles.
\end{abstract}

\section{Introduction.}

La musculature striée des animaux est un ensemble composé d'un nombre important d'éléments, de masse et de forme très variables. Cette diversité s'explique par la très grande variété des fonctions imparties à chacun des muscles dans la locomotion et le maintien de la stature.

La variabilité enregistrée dans les masses - au sein d'un même animal - est un aspect important de l'état du développement musculaire qui est intéressant à considérer à plusieurs titres.

En premier lieu, la variabilité existant entre animaux dans la distribution de la musculature intéresse directement la physiologie de la croissance et du développement musculaire dans la mesure où les équilibres constatés entre muscles résul-

(1) Adresse permanente : Department of Animal Production, Faculty of Agriculture, Ain Shams University, Shoubra El-Kheima, Cairo, Egypt. 
tent des lois propres au développement de chacun, au sein de l'ensemble corporel. L'analyse des interrelations existant entre muscles doit apporter à l'étude de la croissance musculaire un éclairage particulier permettant de dégager la nature et l'ampleur des facteurs favorables, et celles des contraintes, qui interviennent dans le déroulement du processus de croissance musculaire relative de chacun des éléments.

Sur un autre plan, dans le cas des espèces domestiques élevées pour la production de leur chair, la distribution de la musculature conditionne, en partie, l'intérêt des animaux pour leur exploitation en boucherie.

Il existe, en effet, entre muscles, des différences dans la composition et la structure microanatomique qui entraînent des différences notables au niveau de la qualité organoleptique et, pour cette raison, la répartition de la musculature chez un animal donné détermine largement sa valeur d'utilisation.

De nombreuses études ont déjà été consacrées à la répartition de la musculature, soit dans l'ensemble du corps, soit dans des régions anatomiques particulières dans différentes espèces, principalement à des fins zootechniques pour apprécier la valeur des animaux pour la production de viande chez les bovins : (Dumont, Le Guelte et Arnoux, 1961 ; Butterfield, 1965 ; Butterfield et Johnson, 1968; Kauffman et al., 1973; Mukhoty et Berg, 1973; Charles et Johnson, 1976 ; Kempster, Cuthbertson et Smith, 1976; Bergström, 1978 ; Colomer-Rocher, Bass et Johnson, 1980), chez les porcins (Dumont, Schmitt et Roy, 1969 ; Richmond et Berg, 1971 ; Kauffman et al., 1973 ; Davies, 1974 ; Dumont et Roy, 1975 ; Goenaga et Carden, 1979), chez les buffles (Charles et Johnson, 1972 ; Abdallah, Mahallawi et Latif, 1982), chez le poulet (Rouvier et Ricard, 1967) et chez les caprins (Wilson, 1958 ; Gaili, 1976 ; Owen et al., 1978 ; Anous, 1980 ; Owen et al., 1983 ; Gaili et Ali, 1985).

Des études plus orientées sur la physiologie du développement musculaire ont été réalisées chez la grenouille (Stelt et Klijn, 1975) ou l'étourneau (Klijn, 1976).

Dans le cas des ovins, depuis les premiers travaux de Hammond (1932) la distribution musculaire a été envisagée essentiellement dans ses implications zootechniques en considérant diverses races (Hammond, 1932 ; Pálsson, 1940 ; Boccard et Dumont, 1960 ; Boccard et al., 1961 ; Fourie, 1965 ; Boccard et Dumont, 1973 ; Jackson et Mansour, 1974 ; Jury, Fourie et Kirton, 1977 ; Anous, 1980 ; Taylor, Mason et McClelland, 1980 ; Thorgeirsson, 1981 ; Teyssier et Prud'hon, 1982 ; Butterfield et al., 1983 ; Butler-Hogg et Whelehan, 1984 ; Butterfield et al., 1984). Mais dans cette espèce, comme dans les autres, on manque d'information sur l'amplitude de la variation rencontrée entre individus présentant des types morphologiques très différents et chez lesquels on sait maintenant qu'il existe des contraintes particulières du développement musculaire en liaison avec la masse et les dimensions des supports osseux (Anous, 1986a).

Le présent travail rapporte les résultats obtenus dans une étude entreprise chez les ovins en considérant précisément la distribution musculaire au niveau du membre postérieur dans un ensemble d'agneaux des deux sexes représentatifs de la variation existant dans les caractères morphoanatomiques de cette région anatomique et considérés à des états de développement corporel comparables. 
Matériel et méthodes.

Animaux. - L'étude porte sur 88 agneaux répartis en 2 groupes selon le sexe (36 femelles et 52 mâles).

- Pour une part $(N=28)$, des animaux de différents types ont été choisis vivants dans un grand abattoir spécialisé en ovins de manière à constituer un échantillon représentatif des cinq classes de conformation définies dans le système officiel de classement des carcasses de la CEE (*).

- Le restant des animaux $(N=60)$ a été choisi dans des élevages de race pure essentiellement pour constituer des groupes de 6 agneaux 13 mâles et 3 femelles) des races suivantes: Charolaise, lle-de-France, Rava, Romanov, Solognote, Southdown, Suffolk, Tarasconnais, Texel. En plus, on a retenu trois mâles de race Charmoise, deux de race Vendéenne et un de race Rouge-del'Ouest, choisis parmi les agneaux participant au concours d'ovins abattus du concours général agricole 1984 et présentant les meilleures conformations des concurrents de cette épreuve.

Après $24 \mathrm{~h}$ de refroidissement après l'abattage, les carcasses des animaux étaient pesées et la séparation de l'ensemble des membres postérieurs de la carcasse entière opérée par section transversale de la colonne vertébrale entre la $5^{e}$ et la $6^{e}$ vertèbre lombaire. Le poids moyen de carcasse froide a été de $18,9 \pm 3,1 \mathrm{~kg}$.

La partie postérieure était ensuite coupée en deux dans le sens longitudinal par section de l'os pubis et fente de la colonne vertébrale dans le plan sagittal et conservée au froid $\left(-1,+3^{\circ} \mathrm{C}\right)$ jusqu'à la dissection qui s'effectuait dans les 3 jours suivants.

La dissection du membre postérieur droit a été conduite selon les règles classiques de la dissection, en salle réfrigérée à $+10^{\circ} \mathrm{C}$, par séparation individuelle des différents muscles et os et de l'ensemble des graisses. La masse des muscles, pesée sur balance Mettler $K 7$ à $0,025 \mathrm{~g}$ près, était enregistrée au fur et à mesure de leur excision. L'ordre de prélèvement des muscles a été le même pour tous les animaux. L'identification des muscles a été basée sur les ouvrages de Barone (1980), Bénévent (1968), May (1964) et Getty (1975).

A cause des difficultés rencontrées dans la dissection, pour certains muscles, et selon Getty (1975) et May (1964), on a considéré la somme des masses des muscles Tibialis caudalis, Flexor digitorum pedis lateralis et Flexor digitorum pedis medialis comme représentant la masse d'un seul groupe musculaire dénommé Flexor digitorum profundus. On a également regroupé en un seul ensemble les masses des muscles Obturatorius externus et internus.

Les variables musculaires considérées dans cette étude sont les masses des muscles: Tensor fasciae latae (TF), Gluteobiceps (GB), Semitendinosus (ST), Gluteus medius (GM), Gluteus accessorius (GA), Gluteus profundus (GP)

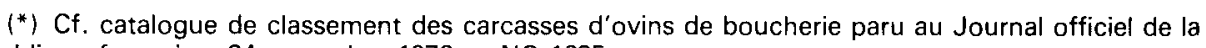
République française, 24 novembre 1976 p. NC 1635. 
Sartorius (SA), Gracilis (GR), Semimembranosus (SM), Adductores (AD), Quadratus femoris (QF), Pectineus (PE), Gemelli (GE), Vastus lateralis (VL), Rectus femoris (RF), Vastus medialis (VM), Vastus intermedius (VI), Somme des muscles Obturatorius (Obturatorius externus + Obturatorius internus) (OB), Popliteus (PO), Flexor digitorium superficialis (FS), Soleus (SO), Tibialis cranialis (TC), Extensor digitorum lateralis (ED), Peroneus tertius (PT), Extensor digiti I longus (LE), Peroneus longus (PL), Flexor digitorum profundus (FP), Gastrocnemius (Caput laterale) (LG), Gastrocnemius (caput mediale) (MG) et Extensor digitorum longus (EL).

La masse de l'ensemble des 30 muscles représente ici la masse totale des muscles du membre postérieur (MT) en $\mathrm{g}$.

Traitement statistique. - Le traitement statistique a été conduit en trois étapes.

1) Dans un premier temps sur l'ensemble des 30 variables, on a calculé, dans chaque sexe :

- la valeur moyenne, l'écart type, le coefficient de variation de chaque variable:

- les corrélations $r_{i i}$ de chacun des muscles avec les autres ;

- la corrélation $r_{M T i}$ entre la masse d'un muscle $m_{i}$ et celle de l'ensemble de la musculature du membre postérieur MT.

Ensuite on a analysé les interrelations entre variables par la méthode d'analyse multidimensionnelle des données centrées (Lefebvre, 1976). Dans cette méthode la projection simultanée des variables et des observations présente un grand intérêt pour l'interprétation comme l'ont montré, par exemple, les travaux de Dumont (1983) ou Dumont et Boulleau (1984). La projection d'un individu sur un axe donné est déterminée par la valeur des différents rapports des variables. En tenant compte principalement des variables les plus discriminantes, parce que les plus opposées sur un axe donné, on peut facilement structurer la population des observations et en déduire les rapports de variables les plus intéressantes pour décrire - en terme d'interrelation entre variables - I'organisation qui y prévaut.

2) Compte tenu des résultats de la première analyse des données centrées on a procédé à une seconde analyse de ce type sur un ensemble plus restreint de variables $(N=24)$ en éliminant du traitement les variables les plus discriminantes dans la première analyse, à savoir SO, QF, SA, VM, ED et GE.

3) Une troisième analyse, par la méthode des données centrées, a été réalisée sur l'ensemble des muscles les plus lourds pour affiner l'étude des interrelations entre muscles les plus importants (TF, GB, ST, GM, SM, AD, VL, RF, VI, LG et FP).

\section{Résultats.}

Les résultats globaux figurent aux tableaux 1 à $4:-$ Valeur moyenne, écart type et coefficient de variation pour chaque muscle (tabl. 1); - Corrélations entre muscles (rij) (tabl. 2); - Corrélations de la masse des muscles avec la masse totale des muscles $\left(r_{M{ }_{T i}}\right)$ du membre (tabl. 3).

Le tableau 4 indique la part de la variation expliquée par les axes dans les trois analyses des données centrées et précise la nature des variables les plus discriminantes. 
Pour l'ensemble des 30 muscles les projections des variables dans les plans définis par les axes 1 et 2 et par les axes 1 et 3 sont représentées dans les figures 3 chez les mâles et 4 chez les femelles. Les mêmes projections sont illustrées pour l'ensemble des 24 muscles par les figures 5 et 6 . Enfin les figures 7 et 8 présentent les projections dans le cas de l'ensemble des onze muscles les plus lourds.

\section{Variabilité des muscles.}

Dans les deux sexes, la masse des muscles varie considérablement. Entre le muscle le plus lourd (GB) et le plus léger (SO) le rapport des moyennes des masses est de 178 chez les mâles et de $166 \mathrm{chez}$ les femelles. L'analyse de la distribution des masses montre que 21 muscles sur 30, dans les deux sexes, ont une masse inférieure à la masse musculaire moyenne (MT/30) (qui est de $60,9 \mathrm{~g}$ chez les mâles et de 56,8 g chez les femelles). Dans l'ordre des masses croissantes, la

TABLEAU 1

Moyenne et écart type (en g) et coefficient de variation (en \%) des masses des muscles individuels et du muscle total du membre postérieur.

\begin{tabular}{|c|c|c|c|c|c|c|}
\hline \multirow{2}{*}{ Muscles } & \multicolumn{3}{|c|}{ Mâles } & \multicolumn{3}{|c|}{ Femelles } \\
\hline & $\bar{x}$ & s & C.V. \% & $\bar{x}$ & $\mathrm{~s}$ & C.V. \% \\
\hline so & 1,52 & 0,67 & 43,91 & 1,56 & 0,65 & 41,93 \\
\hline $\mathrm{GE}$ & 3,19 & 0,86 & 26,90 & 2,79 & 0,63 & 22,71 \\
\hline ED & 3,51 & 0,94 & 26,74 & 3,53 & 0,71 & 20,18 \\
\hline $\mathrm{OF}$ & 3,66 & 1,17 & 32,01 & 3,25 & 1,26 & 38,89 \\
\hline $\mathrm{PL}$ & 6,29 & 1,70 & 27,02 & 5,55 & 1,32 & 23,86 \\
\hline EL & 7,66 & 1,47 & 19,27 & 6,82 & 1,55 & 22,75 \\
\hline $\mathrm{LE}$ & 8,47 & 1,71 & 20,22 & 7,96 & 1,62 & 20,30 \\
\hline TC & 9,17 & 1,96 & 21,33 & 9,02 & 1,88 & 20,86 \\
\hline SA & 10,03 & 3,13 & 31,16 & 8,68 & 2,80 & 32,20 \\
\hline PO & 16,69 & 2,66 & 15,96 & 14,52 & 2,43 & 16,77 \\
\hline PT & 19,13 & 3,51 & 18,36 & 16,40 & 3,25 & 19,84 \\
\hline GP & 20,09 & 4,90 & 24,37 & 17,05 & 4,11 & 24,13 \\
\hline $\mathrm{GA}$ & 28,43 & 7,26 & 25,54 & 25,36 & 7,25 & 28,60 \\
\hline $\mathrm{OB}$ & 30,04 & 5,00 & 16,64 & 28,46 & 4,95 & 17,39 \\
\hline PE & 32,16 & 7,00 & 21,75 & 29,84 & 5,97 & 20,01 \\
\hline FS & 37,25 & 7,33 & 19,67 & 33,63 & 7,63 & 22,69 \\
\hline VM & 40,97 & 13,89 & 33,89 & 39,75 & 11,27 & 28,35 \\
\hline MG & 41,38 & 7,17 & 17,33 & 38,88 & 6,32 & 16,26 \\
\hline GR & 50,82 & 12,04 & 23,69 & 43,89 & 10,99 & 25,04 \\
\hline TF & 56,32 & 10,95 & 19,44 & 53,07 & 10,67 & 20,10 \\
\hline LG & 57,76 & 11,14 & 19,28 & 52,45 & 10,67 & 20,35 \\
\hline VI & 67,15 & 11,24 & 16,74 & 59,02 & 12,03 & 20,38 \\
\hline FP & 68,70 & 11,61 & 16,90 & 62,30 & 11,76 & 18,87 \\
\hline ST & 102,86 & 19,51 & 18,97 & 95,36 & 18,36 & 19,26 \\
\hline$A D$ & 117,70 & 20,42 & 17,35 & 114,13 & 21,95 & 19,23 \\
\hline $\mathrm{RF}$ & 130,06 & 19,31 & 14,84 & 122,73 & 18,44 & 15,02 \\
\hline VL & 138,83 & 22,42 & 16,15 & 135,35 & 20,18 & 14,91 \\
\hline GM & 180,01 & 30,29 & 16.83 & 169,65 & 33,69 & 19,86 \\
\hline SM & 267,31 & 48,42 & 18,11 & 251,19 & 44,59 & 17,75 \\
\hline $\mathrm{GB}$ & 270,60 & 48,67 & 17,99 & 259,30 & 49,00 & 18,90 \\
\hline Ensemble des & & & & & & \\
\hline $\begin{array}{l}\text { muscles du } \\
\text { membre } \\
\text { postérieur }\end{array}$ & 1827,70 & 277,90 & 15,20 & 1711,50 & 218,20 & 16,48 \\
\hline
\end{tabular}


masse du muscle classé $10^{\mathrm{e}}$ ne représente chez les mâles que $0,91 \%$ de la musculature totale du membre et, chez les femelles, $0,85 \%$ de la musculature totale. Celle-ci est donc composée, pour l'essentiel, d'un fort pourcentage de muscles de masse peu importante $(<$ à $50 \mathrm{~g})$ et d'un petit nombre de muscles relativement lourds ou très lourds ( $\geq$ à $100 \mathrm{~g}$ ). Ces derniers peuvent être, sur le strict plan de l'importance pondérale, considérés comme majeurs, par opposition aux autres et spécialement par rapport à ceux dont la masse est inférieure à $10 \mathrm{~g}$ et que nous considérerons, au plan de la masse, comme mineurs (tabl. 1).

Les coefficients de variation de la masse des muscles varient d'un muscle à l'autre et vont de 14,84 à $43,91 \%$ chez les mâles respectivement pour les muscles RF et SO et de 14,91 à $41,93 \%$ chez les femelles pour les muscles VL et SO. Ils sont, dans les deux sexes, voisins pour leur valeur moyenne $121,95 \%$ chez les mâles et 22,25 \% chez les femelles) et pour leur variabilité (CV de 29,98\% chez les mâles et de $28,30 \%$ chez les femelles) (tabl. 1).

D'une façon générale, la variabilité des muscles les plus lourds est plus faible et réciproquement comme le montre, par exemple, dans les deux sexes, le classement comparé des rangs des muscles selon la masse croissante et le coefficient de variation décroissant.

Toutefois, au travers de cette influence de la masse sur la variabilité, on peut déceler une influence spécifique de la nature du muscle que révèlent les graphiques de la variation du classement selon le coefficient de variation par rapport au classement selon la masse (fig. 1). Il apparaît aussi que les muscles peuvent se classer en trois groupes selon que leur variabilité est soit trop faible par rapport à leur masse, soit trop forte, soit d'un niveau moyen correspondant à l'effet général de la masse sur la variabilité. Le premier cas se retrouve dans les deux sexes pour les muscles $P O, O B$ et $P T$ et spécialement chez les mâles pour $E L$, et chez les femelles pour MG, GE et ED. Le second cas s'observe dans les deux sexes pour les muscles VM et GR et chez les mâles seulement pour SM et les femelles pour $\mathrm{GM}, \mathrm{VI}$ et GA. II semble donc que la relation variabilité-masse ne soit pas identique dans les deux sexes.

\section{Relations entre muscles.}

1) Corrélations simples.

On observe une grande varibilité dans la valeur des coefficients de corrélation entre les masses des différents muscles. Sur l'ensemble des 435 relations on observe qu'un certain nombre ne sont pas significatives (au seuil $5 \%$ ) et ne seront donc pas représentées (tabl. 2). Elles représentent $17,5 \%$ des corrélations chez les mâles et $19,1 \%$ chez les femelles. Elles concernent essentiellement les relations faisant intervenir les muscles QF, GE, ED, GP avec les autres. Les corrélations qui ne sont significatives qu'au seuil $5 \%$ représentent $7,1 \%$ de l'ensemble chez les mâles et $9,4 \%$ chez les femelles. Elles impliquent principalement chez les mâles les relations mettant en jeu les muscles SO, SA et GE avec les autres muscles et chez les femelles celles concernant les relations de SO, QF et VM.

Les autres corrélations $(75,4 \%$ chez les mâles et $71,5 \%$ chez les femelles) sont hautement significatives au seuil $1 \%$. Parmi elles la variabilité est impor- 

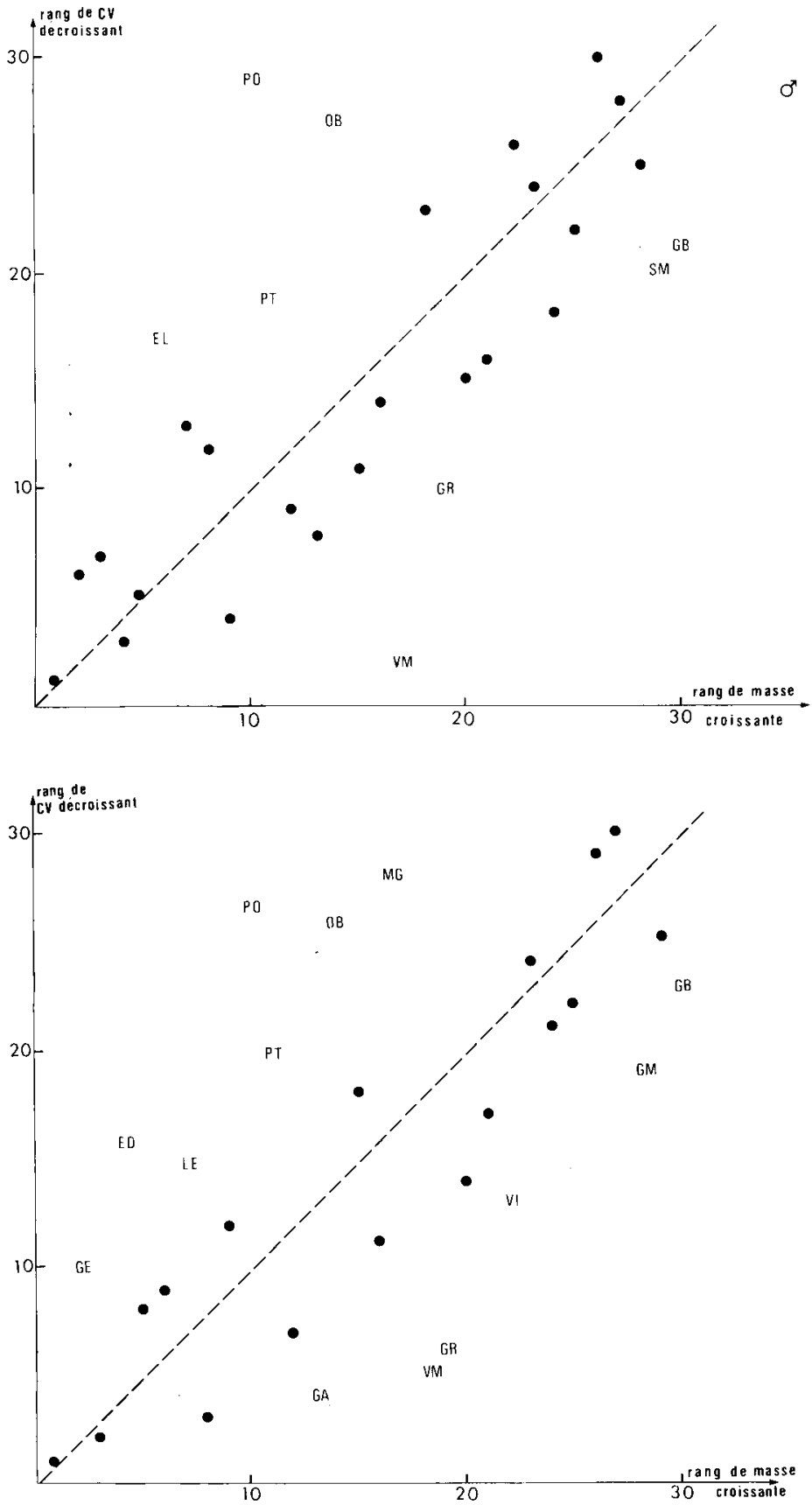

FIG. 1. - Variation du classement des muscles selon le coefficient de variation, par rapport à leur classement selon la masse chez les máles (au-dessus) et les femelles (au-dessous). Les ronds noirs (-) concernent les muscles dont le coefficient de variation peut être considéré comme correspondant à l'effet général de la masse sur la variabilité. 
TABLEAU 2

Corrélations entre masses des muscles individuels.

\begin{tabular}{|c|c|c|c|c|c|c|c|c|c|c|c|c|c|c|c|}
\hline$\sigma$ & TF & GB & ST & GM & GA & GP & SA & GR & SM & $A D$ & $\mathrm{QF}$ & GE & PE & VL & RF \\
\hline Q & & & & & & & & & & & & & & & \\
\hline TF & & 0,74 & 0,77 & 0,68 & 0,65 & 0,48 & 0,38 & 0,67 & 0,74 & 0,67 & - & 0,39 & 0,56 & 0,74 & 0,71 \\
\hline GB & 0,80 & & 0,84 & 0,89 & 0,67 & 0,53 & 0,58 & 0,63 & 0,91 & 0,83 & - & & 0,75 & 0,85 & 0,81 \\
\hline ST & 0,75 & 0,90 & & 0,80 & 0,79 & 0,58 & 0,44 & 0,73 & 0,90 & 0,89 & - & 0,35 & 0,70 & 0,85 & \\
\hline $\mathrm{GM}$ & 0,68 & 0,89 & 0,82 & & 0,53 & 0.44 & 0,41 & 0,60 & 0,89 & 0,78 & - & 0,31 & 0,69 & 0,83 & 0,76 \\
\hline GA & 0,66 & 0,70 & 0,62 & 0,62 & & 0,47 & 0,32 & 0,59 & 0,73 & 0,73 & - & - & 0,51 & 0,65 & 0,63 \\
\hline GP & 0,54 & 0,61 & 0,65 & 0,69 & 0,69 & & 0,35 & 0,40 & 0,54 & 0,51 & - & - & 0,59 & 0,56 & 0,49 \\
\hline SA & 0,66 & 0,67 & 0,61 & 0,54 & 0,44 & 0,41 & & 0,41 & 0,44 & 0,43 & - & - & 0,65 & 0,34 & 0,37 \\
\hline GR & 0,83 & 0,88 & 0,81 & 0,80 & 0,73 & 0,69 & 0,78 & & 0,66 & 0,66 & - & 0,43 & 0,41 & 0,59 & 0,51 \\
\hline SM & 0,79 & 0,92 & 0,85 & 0,84 & 0,72 & 0,65 & 0,56 & 0,82 & & 0,90 & - & - & 0,77 & 0,88 & 0,77 \\
\hline$A D$ & 0,75 & 0,85 & 0,90 & 0,78 & 0,70 & 0,72 & 0,61 & 0,83 & 0,83 & & - & - & 0,76 & 0,82 & 0,73 \\
\hline QF & 0,35 & - & - & 0,35 & - & 0,40 & - & 0,34 & - & 0,40 & & 0,45 & - & - & - \\
\hline GE & - & - & - & - & 0,35 & - & - & - & - & - & 0,38 & & - & 0,40 & 0,34 \\
\hline PE & 0,74 & 0,85 & 0,81 & 0,79 & 0,67 & 0,73 & 0,70 & 0,82 & 0,79 & 0,86 & 0,34 & - & & 0,73 & 0,70 \\
\hline VL & 0,70 & 0,80 & 0,81 & 0,81 & 0,57 & 0,72 & 0,57 & 0,79 & 0,75 & 0,80 & 0,44 & - & 0,72 & & 0,89 \\
\hline RF & 0,69 & 0,66 & 0,68 & 0,76 & 0,60 & 0,76 & 0,45 & 0,65 & 0,73 & 0,73 & 0,44 & 0,37 & 0,68 & 0,77 & \\
\hline VM & - & 0,35 & - & 0,56 & - & 0,54 & - & 0,37 & 0,39 & - & - & - & 0,42 & 0,48 & 0,55 \\
\hline VI & 0,61 & 0,66 & 0,69 & 0,65 & 0,58 & 0,59 & 0,47 & 0,62 & 0,65 & 0,65 & 0,56 & - & 0,62 & 0,66 & 0,58 \\
\hline LG & 0,71 & 0,87 & 0,75 & 0,79 & 0,68 & 0,59 & 0,58 & 0,80 & 0,88 & 0,72 & - & - & 0,78 & 0,67 & 0,65 \\
\hline MG & 0,65 & 0,88 & 0,77 & 0,83 & 0,63 & 0,49 & 0,49 & 0,69 & 0,84 & 0,69 & - & - & 0,73 & 0,68 & 0,68 \\
\hline$O B$ & 0,36 & 0,55 & 0,59 & 0,67 & 0,55 & 0,70 & 0,37 & 0,56 & 0,57 & 0,55 & - & - & 0,55 & 0,63 & 0,65 \\
\hline FS & 0,68 & 0,81 & 0,70 & 0,78 & 0,71 & 0,63 & 0,51 & 0,79 & 0,84 & 0,67 & - & - & 0,73 & 0,71 & 0,61 \\
\hline so & 0,48 & - & - & 0,35 & 0,48 & 0,42 & - & 0,36 & 0,37 & 0,42 & 0,36 & 0,43 & 0,35 & - & 0,35 \\
\hline TC & 0,63 & 0,75 & 0,73 & 0,68 & 0,62 & 0,50 & 0,75 & 0,79 & 0,69 & 0,68 & - & - & 0,60 & 0,72 & 0,52 \\
\hline EL & 0,64 & 0,82 & 0,73 & 0,86 & 0,79 & 0,69 & 0,49 & 0,75 & 0,84 & 0,77 & 0,36 & - & 0,74 & 0,76 & 0,78 \\
\hline ED & - & - & 0,34 & - & - & 0,37 & - & - & - & - & - & - & - & 0,46 & 0,43 \\
\hline PT & 0,70 & 0,78 & 0,75 & 0,71 & 0,60 & 0,45 & 0,63 & 0,82 & 0,75 & 0,66 & - & - & 0,65 & 0,67 & 0,51 \\
\hline LE & 0,62 & 0,82 & 0,75 & 0,86 & 0,67 & 0,66 & 0,56 & 0,73 & 0,8 & 0,7 & - & - & 0,74 & 0,81 & 0,67 \\
\hline $\mathrm{PL}$ & 0,47 & 0,70 & 0,51 & 0,61 & 0,64 & 0,43 & 0,4 & 0,59 & 0,63 & 0,5 & - & - & 0,57 & 0,50 & 0,42 \\
\hline $\mathrm{PO}$ & 0,57 & 0,74 & 0,74 & 0,67 & 0,60 & 0,5 & 0,5 & 0,72 & 0,72 & 0,7 & - & - & 0,68 & 0,73 & 0,50 \\
\hline FP & 0,68 & 0,82 & 0,71 & 0,79 & 0,80 & 0,50 & 0,46 & 0,71 & 0,85 & 0,68 & -_ & - & 0,64 & 0,64 & 0,64 \\
\hline
\end{tabular}

NB. Les corrélations non significatives sont représentées par un tiret $(-)$.

tante, et en fait, un faible nombre de corrélations sont finalement d'un niveau très élevé : seuls 25 chez les mâles et 30 chez les femelles sont supérieurs à $+0,83$.

En considérant la valeur moyenne des corrélations des muscles par région et sous-région on constate que les moyennes des corrélations sont, également, très différentes et que c'est au niveau de la sous-région fémorale caudale que les moyennes sont les plus fortes (respectivement $+0,88$ pour les mâles et $+0,89$ pour les femelles) en raison des fortes corrélations entre les trois muscles qui la composent (GB, SM et ST). Pour les autres sous-régions les corrélations entre muscles ne sont assez élevées $(r>+0,70)$ que pour un nombre limité de liaisons (e.g. VL et RF, AD et PE, MG et $L G, L G$ et $F S, M G$ et $F S, T C$ et $L E$, EL et $L E, F P$ et $L G$ ). Dans le cas des muscles les plus lourds (GB, SM, GM, VL, RF et AD) les corrélations sont, globalement, relativement élevées.

Les corrélations entre la masse de chacun des muscles et la masse totale des muscles $\left(r_{M T i}\right)$ varient considérablement, d'un muscle à l'autre, dans les deux sexes. Chez les mâles, 22 muscles ont avec MT une corrélation supérieure à 0,7 
TABLEAU 2

(suite)

\begin{tabular}{|c|c|c|c|c|c|c|c|c|c|c|c|c|c|c|}
\hline VM & VI & LG & MG & OB & FS & so & TC & EL & ED & PT & LE & PL & PO & FP \\
\hline 0,49 & 0,48 & 0,57 & 0,56 & 0,51 & 0,73 & 0,45 & 0,59 & 0,68 & - & 0,66 & 0,56 & 0,54 & 0,66 & 0,74 \\
\hline 0,64 & 0,33 & 0,83 & 0,84 & 0,43 & 0,78 & 0,40 & 0,75 & 0,75 & - & 0,77 & 0,74 & 0,49 & 0,65 & 0,83 \\
\hline 0,56 & 0,50 & 0,77 & 0,75 & 0,49 & 0,81 & 0,37 & 0,70 & 0,76 & - & 0,72 & 0,65 & 0,54 & 0,66 & 0,81 \\
\hline 0,70 & 0,28 & 0,82 & 0,82 & 0,43 & 0,75 & 0,38 & 0,67 & 0,70 & - & 0,66 & 0,64 & 0,44 & 0,53 & 0,76 \\
\hline 0,42 & 0,43 & 0,62 & 0,62 & 0,45 & 0,68 & 0,30 & 0,61 & 0,64 & - & 0,62 & 0,65 & 0,33 & 0,57 & 0,70 \\
\hline 0,36 & 0,50 & 0,49 & 0,41 & - & 0,54 & 0,29 & 0,55 & 0,63 & - & 0,53 & 0,58 & 0,49 & 0,50 & 0,59 \\
\hline- & - & 0,54 & 0,46 & - & 0,38 & 0,30 & 0,47 & 0,49 & - & 0,59 & 0,31 & 0,35 & 0,32 & 0,44 \\
\hline 0,30 & 0,45 & 0,52 & 0,53 & $\cdots$ & 0,52 & 0,43 & 0,61 & 0,59 & - & 0,63 & 0,41 & 0,43 & 0,54 & 0,58 \\
\hline 0,67 & 0,36 & 0,85 & 0,83 & 0,43 & 0,84 & 0,38 & 0,74 & 0,76 & - & 0,69 & 0,75 & 0,49 & 0,68 & 0,82 \\
\hline 0,59 & 0,45 & 0,77 & 0,75 & 0,32 & 0,83 & 0,45 & 0,73 & 0,76 & - & 0,66 & 0,73 & 0,48 & 0,69 & 0,81 \\
\hline- & 0,36 & - & - & - & - & 0,41 & - & - & - & - & - & 0,40 & 0,28 & \\
\hline- & 0,43 & - & - & - & - & 0,29 & 0,29 & - & - & - & - & 0,38 & & 0,30 \\
\hline 0,59 & 0,33 & 0,75 & 0,63 & 0,48 & 0,74 & 0,33 & 0,67 & 0,69 & - & 0,61 & 0,61 & 0,55 & 0,62 & 0,70 \\
\hline 0,70 & 0,50 & 0,76 & 0,76 & 0,53 & 0,81 & 0,40 & 0,79 & 0,75 & - & 0,64 & 0,73 & 0,63 & 0,68 & 0,83 \\
\hline 0,70 & 0,42 & 0,70 & 0,73 & 0,68 & 0,76 & - & 0,67 & 0,69 & 0,32 & 0,61 & 0,66 & 0,58 & 0,65 & 0,78 \\
\hline & - & 0,61 & 0,64 & 0,44 & 0,71 & - & 0,49 & 0,49 & - & 0,45 & 0,60 & 0,51 & 0,58 & 0,63 \\
\hline- & & 0,29 & 0,30 & - & 0,41 & 0,49 & 0,46 & 0,49 & - & 0,41 & 0,38 & 0,42 & 0,48 & 0,48 \\
\hline 0,41 & 0,54 & & 0,91 & 0,49 & 0,85 & 0,35 & 0,70 & 0,76 & - & 0,73 & 0,70 & 0,44 & 0,54 & 0,83 \\
\hline 0,39 & 0,58 & 0,89 & & 0,48 & 0,79 & 0,29 & 0,73 & 0,68 & - & 0,71 & 0,71 & 0,44 & 0,56 & 0,82 \\
\hline 0,42 & 0,47 & 0,40 & 0,48 & & 0,53 & - & 0,35 & 0,46 & 0,35 & 0,36 & 0,37 & 0,41 & 0,41 & 0,55 \\
\hline 0,47 & 0,59 & 0,89 & 0,81 & 0,51 & & 0,45 & 0,66 & 0,76 & - & 0,72 & 0,73 & 0,56 & 0,73 & 0,86 \\
\hline- & 0,58 & - & - & - & - & & 0,35 & 0,51 & - & 0,37 & 0,43 & 0,45 & 0,36 & 0,44 \\
\hline- & 0,58 & 0,62 & 0,59 & 0,62 & 0,62 & 0,35 & & 0,75 & - & 0,65 & 0,72 & 0,50 & 0,62 & 0,78 \\
\hline 0,47 & 0,69 & 0,82 & 0,82 & 0,65 & 0,84 & 0,43 & 0,67 & & - & 0,64 & 0,80 & 0,54 & 0,61 & 0,79 \\
\hline- & 0,38 & - & - & 0,37 & - & - & - & - & & - & - & - & - & - \\
\hline 0,38 & 0,45 & 0,74 & 0,72 & 0,53 & 0,77 & - & 0,77 & 0,69 & - & & 0,59 & 0,41 & 0,63 & 0,81 \\
\hline 0,48 & 0,61 & 0,72 & 0,74 & 0,70 & 0,73 & 0,36 & 0,77 & 0,84 & - & 0,73 & & 0,37 & 0,66 & 0,78 \\
\hline- & 0,39 & 0,62 & 0,57 & 0,34 & 0,60 & . & 0,59 & 0,67 & - & 0,47 & 0,62 & & 0,51 & 0,59 \\
\hline- & 0,57 & 0,60 & 0,53 & 0,60 & 0,72 & 0,39 & 0,65 & 0,72 & - & 0,68 & 0,70 & 0,61 & & 0,71 \\
\hline 0,36 & 0,59 & 0,82 & 0,85 & 0,50 & 0,84 & 0,41 & 0,65 & 0,91 & - & 0,72 & 0,77 & 0,64 & 0,67 & \\
\hline
\end{tabular}

et 4 une corrélation supérieure à 0,9 . Chez les femelles la corrélation est supérieure à 0,7 pour 19 muscles et dépasse 0,91 pour 5 d'entre eux. On peut déduire de ces valeurs que pour une part la variabilité rencontrée au sein de l'échantillon dans le poids des différents muscles est liée à la variabilité de la musculature totale mais qu'une part non négligeable de la variabilité de leur poids est spécifique de leur nature propre (tabl. 3).

En considérant graphiquement (fig. 2) la relation existant entre la valeur de la corrélation $\mathrm{r}_{\mathrm{MT}}$ des différents muscles et leur masse, on peut observer que, globalement, $r_{M T i}$ dépend de la masse dans une forme de liaison qu'il n'a pas été envisagé d'étudier de manière détaillée dans ce travail.

Dans chaque sexe le graphique fait ressortir la position particulière de certains muscles pour lesquels les valeurs de $r_{\mathrm{MTi}}$ sont plus élevées ou moins élevées qu'elles devraient l'être compte tenu de la relation supposée qui vient d'être évoquée. C'est ce qui se passe dans le premier cas, pour les muscles EL, TC, LE, PL, PO, PT, SO dans les deux sexes et en plus pour les femelles pour les muscles SA 
et GP. Dans le second cas paraissent se trouver dans les deux sexes les muscles $V I$ et $V M$ et chez les mâles, en plus, QF, ED, OB et GR.

TABLEAU 3

Valeurs des corrélations $\left(\mathrm{r} M T_{i}\right)$ entre la masse de chacun des muscles $\left(m_{i}\right)$ et la masse totale de muscles (MT) du membre postérieur.

\begin{tabular}{|c|c|c|}
\hline Muscles & Mâles & Femelles \\
\hline so & $+0,44$ & $+0,41$ \\
\hline GE & $+0,32$ & $+0,20$ \\
\hline ED & $+0,13$ & $+0,27$ \\
\hline $\mathrm{QF}$ & $+0,16$ & $+0,36$ \\
\hline $\mathrm{PL}$ & $+0,65$ & $+0,65$ \\
\hline $\mathrm{EL}$ & $+0,82$ & $+0,90$ \\
\hline LE & $+0,79$ & $+0,86$ \\
\hline $\mathrm{TC}$ & $+0,80$ & $+0,76$ \\
\hline SA & $+0,50$ & $+0,65$ \\
\hline PO & $+0,73$ & $+0,77$ \\
\hline $\mathrm{PT}$ & $+0,77$ & $\begin{array}{r}0,79 \\
\end{array}$ \\
\hline GP & $+0,60$ & $+0,75$ \\
\hline $\mathrm{GA}$ & $+0,74$ & $+0,76$ \\
\hline $\mathrm{OB}$ & $+0,53$ & $+0,65$ \\
\hline$P E$ & $+0,80$ & $+0,87$ \\
\hline FS & $+0,88$ & $+0,86$ \\
\hline VM & $+0,70$ & $+0,47$ \\
\hline$M G$ & $+0,86$ & $+0,87$ \\
\hline GR & $+0,70$. & $+0,90$ \\
\hline $\mathrm{TF}$ & $+0,80$ & $+0,83$ \\
\hline LG & $+0,87$ & $+0,88$ \\
\hline $\mathrm{VI}$ & $+0,46$ & $+0,71$ \\
\hline $\mathrm{FP}$ & $+0,90$ & $+0,85$ \\
\hline ST & $+0,93$ & $+0,91$ \\
\hline$A D$ & $+0,91$ & $+0,90$ \\
\hline $\mathrm{RF}$ & $+0,87$ & $+0,81$ \\
\hline $\mathrm{VL}$ & $+0,93$ & $+0,87$ \\
\hline $\mathrm{GM}$ & $+0,91$ & $+0,93$ \\
\hline SM & $+0,96$ & $+0,95$ \\
\hline GB & $+0,95$ & $+0,96$ \\
\hline
\end{tabular}



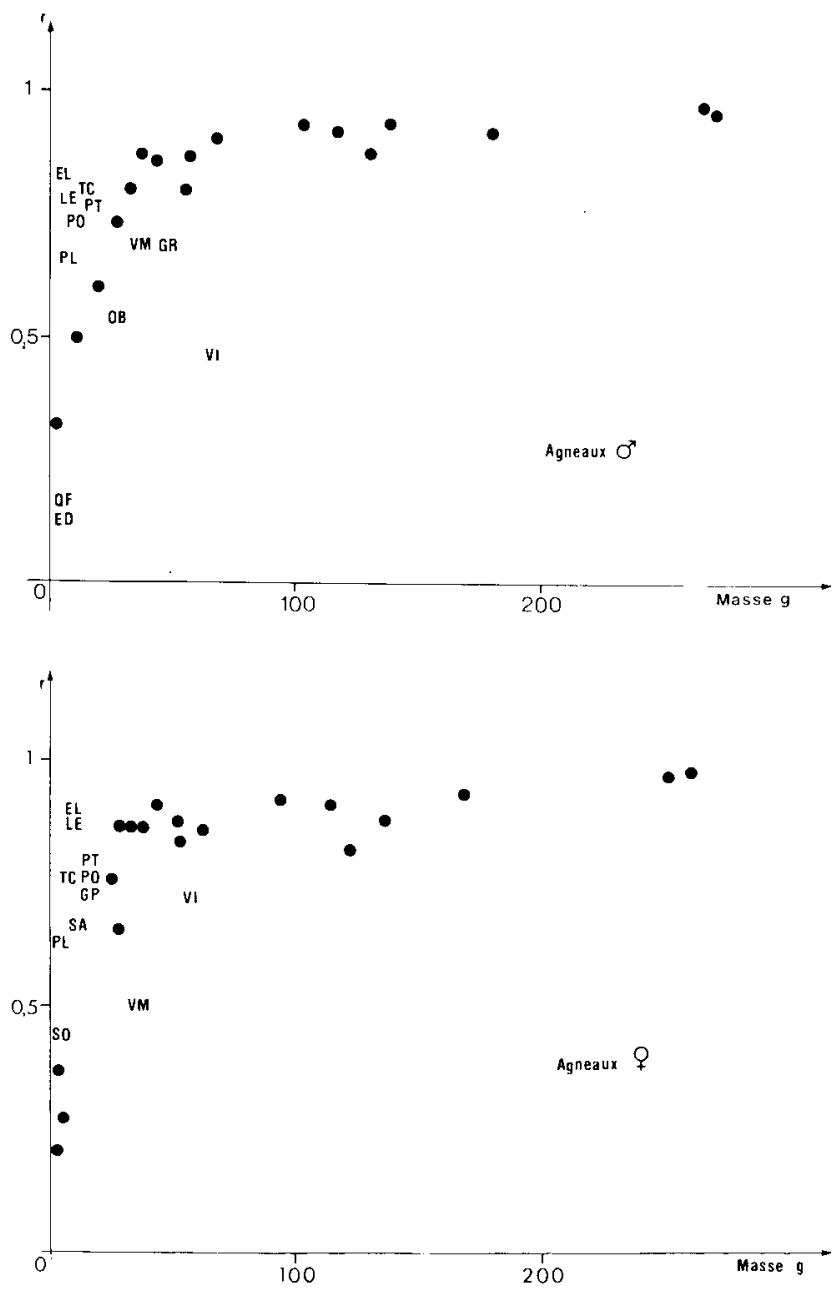

FIG. 2. - Relation entre la valeur de la corrélation $\left(\mathrm{r}_{\mathrm{MTi}}\right)$ des différents muscles et leur masse (mi) chez les mâles (au-dessus) et les femelles (au-dessous).

2) Interrelations entre muscles.

a) Projection de l'ensemble des trente muscles. - La part d'explication par les axes successifs est comparable dans les deux sexes. Sur les six premiers axes on explique environ les trois quarts de la variation de la population (tabl. 4).

La part relativement faible des premiers axes suggère qu'il n'existe pas de variables majeures, hautement discriminantes par rapport aux autres au sein de la population où doivent exister, en fait, pour structurer la population, une série d'équilibres successifs entre variables. II semble donc qu'on ne puisse pas parler d'une distribution uniforme de la musculature et qu'il n'existe pas une homéostasie stricte de la composition musculaire. 


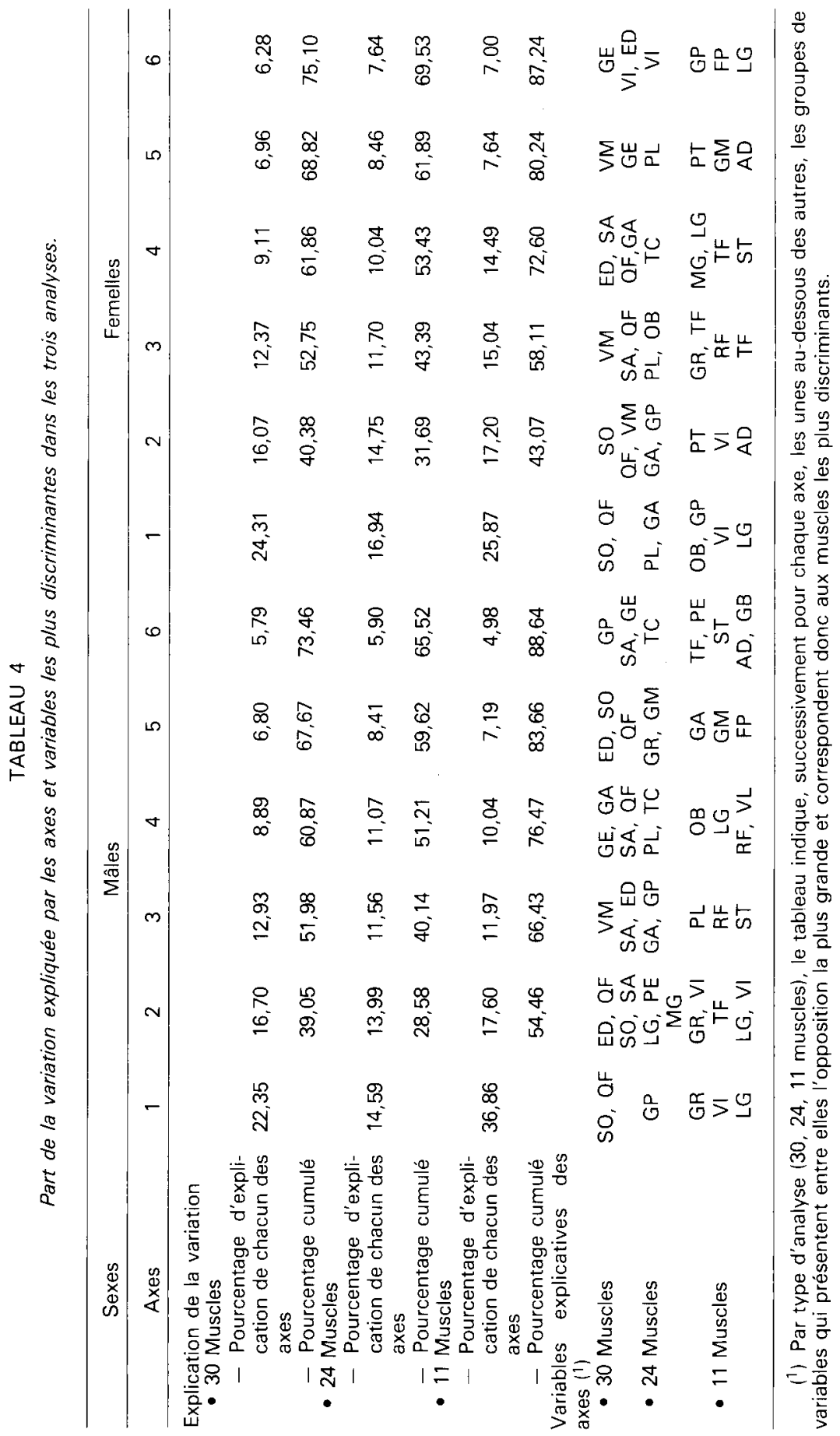


Les variables explicatives des premiers axes ne sont pas rigoureusement les mêmes - dans leur ordre d'importance - dans les deux sexes, mais on remarque toutefois que dans l'espace des trois premiers axes les mêmes variables interviennent pour classer les animaux (à savoir SO, OF, SA et VM) et que sur l'ensemble des 5 premiers axes interviennent en outre comme variables discriminantes communes aux deux sexes ED et GE (fig. 3 et fig. 4).

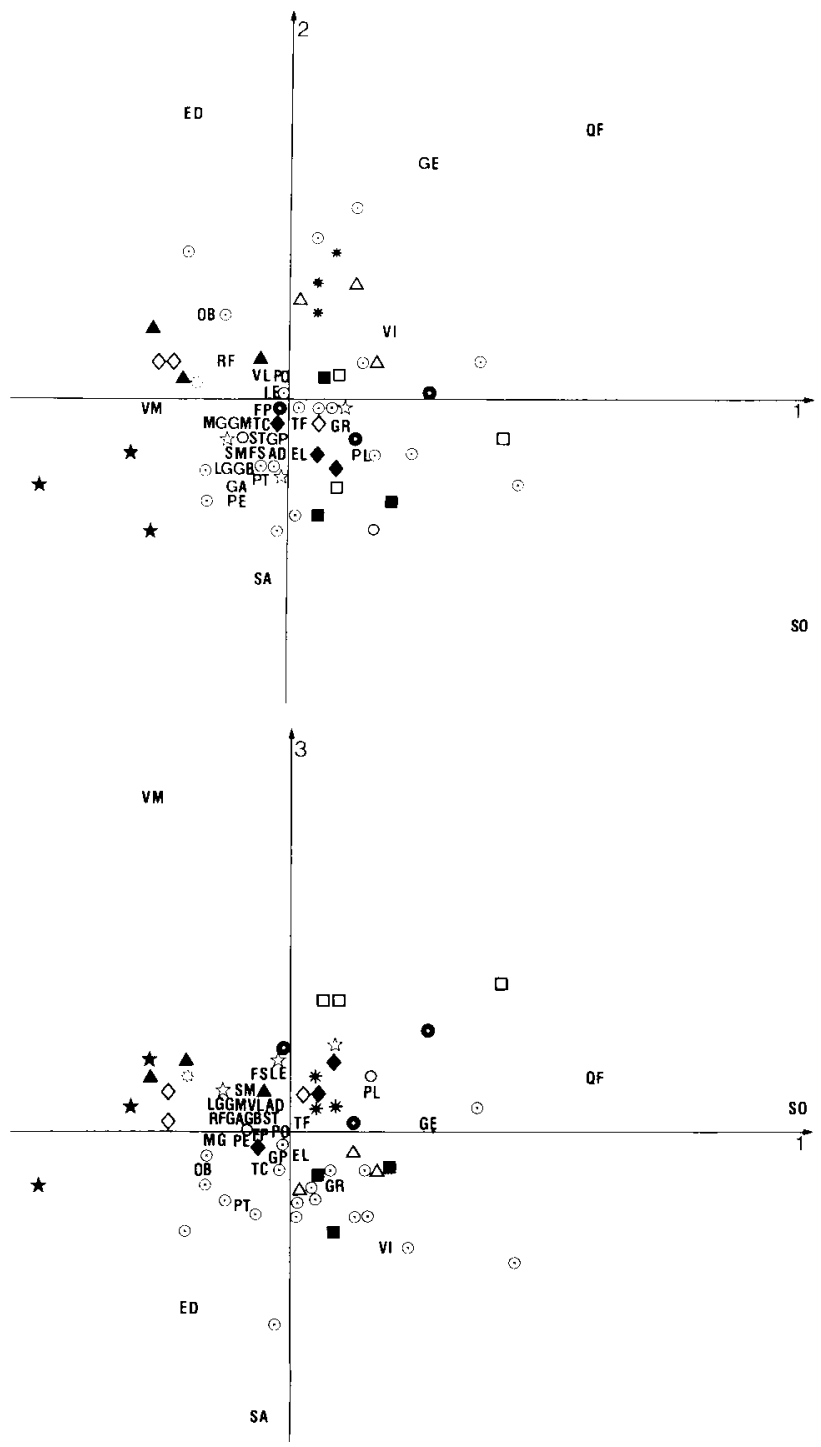

FIG. 3. - Plan de projection de l'ensemble des 30 muscles avec l'ensemble des animaux sur le premier et le deuxieme axe (au-dessus) et le premier et le troisième axe (au-dessous) chez les males. (D) Texel, $(\Delta)$ Solognot, $(\mathbf{\Delta})$, Rava, $(\diamond)$ lle-de-France, $(\bullet)$ Charmois, $(3)$ Rouge-de-l'Ouest, (O) Vendéen, (*) Suffolk, (is) Charolais, (*) Romanov, (৫) Tarasconnais, $(\square)$ Southdown, $(\otimes)$ Agneau croisé. 
Les variables les plus discriminantes de la population sont des variables de masse très faible (SO, ED, GE et QF) ou faible (SA) ou moyenne (VM), et ne présentent que $3 \%$ du MT.

Dans l'espace des trois premiers axes la position des variables les plus discriminantes permet de distinguer nettement des axes de classement des individus

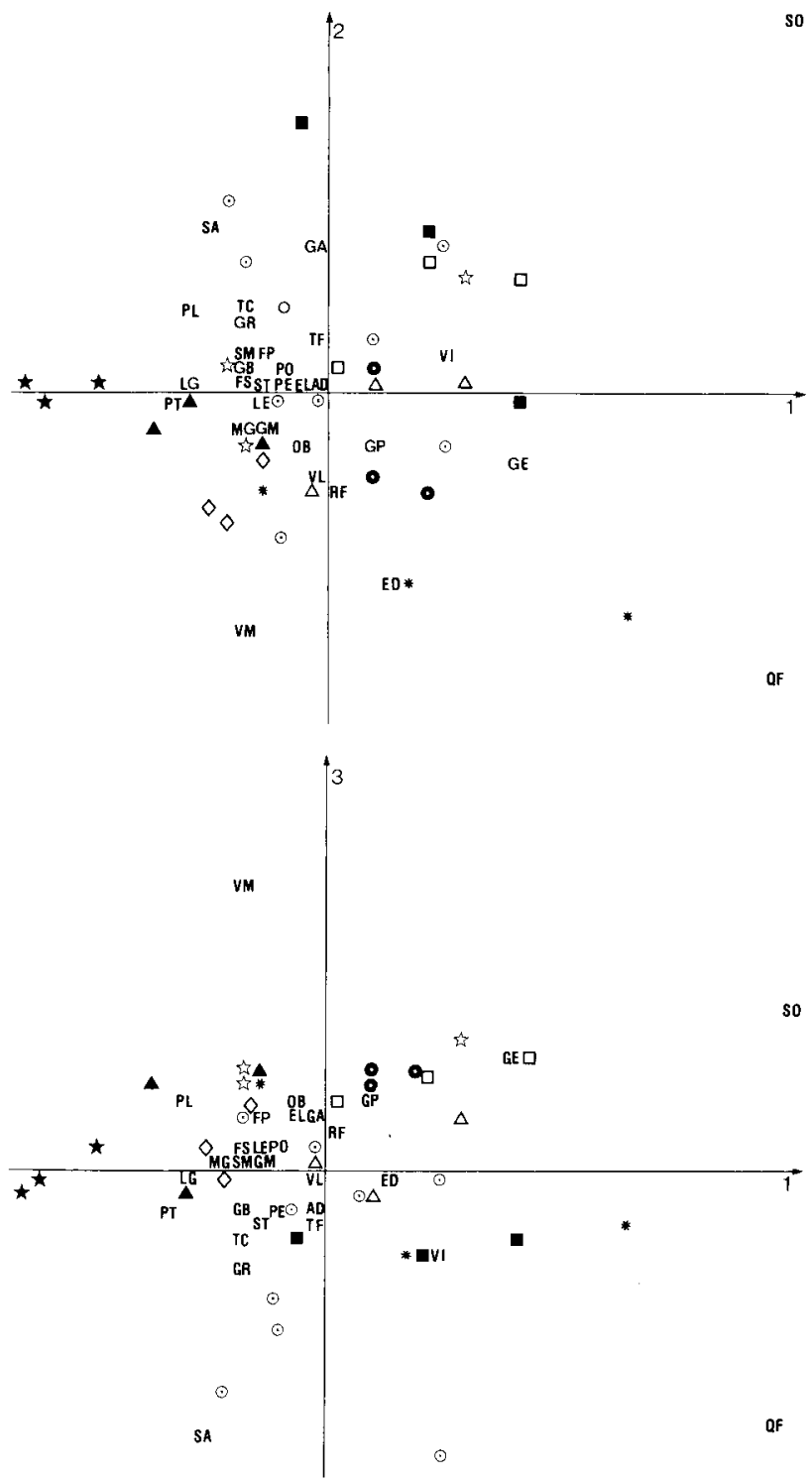

FIG. 4. - Plan de projection de l'ensemble des 30 muscles avec l'ensemble des animaux sur le premier et le deuxième axe (au-dessus) et le premier et le troisième axe (au-dessous) chez les

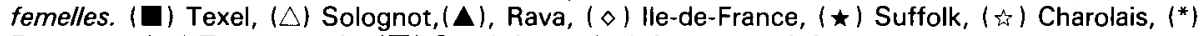
Romanov, (•) Tarasconnais, ( $\square$ ) Southdown, $(\otimes)$ Agneau croisé. 
sur la base de différents rapports, spécialement : VM/QF et VM/SO, également nets dans les deux sexes ; SO/QF, plus net chez les femelles que chez les mâles ; $\mathrm{SA} / \mathrm{QF}$, plus net chez les mâles; et VM/SA, qui correspond à l'axe 3 dans les deux sexes.

L'amplitude de la variation rencontrée dans la population est illustrée par les valeurs des différents rapports enregistrés chez des animaux opposés dans l'espace de projection des trois premiers axes (tabl. 5).

TABLEAU 5

Valeurs des rapports des variables discriminantes.

\begin{tabular}{|c|c|c|c|c|}
\hline \multirow{2}{*}{ Animal $n^{\circ}$} & \multicolumn{4}{|c|}{ Rapport } \\
\hline & VM/SO & $\mathrm{SA} / \mathrm{QF}$ & VM/OF & $V M / S A$ \\
\hline 4 & 10,00 & 3,39 & 6,15 & 1,81 \\
\hline 5 & 17,00 & 1,64 & 7,59 & 4,60 \\
\hline 6 & 17,00 & 6,53 & 8,03 & 1,23 \\
\hline 21 & 52,60 & 2,03 & 8,76 & 4,31 \\
\hline 43 & 107,40 & 4,47 & 16,78 & 6,80 \\
\hline 63 & 62,90 & 11,33 & 41,93 & 3,70 \\
\hline 73 & 30,30 & 1,09 & 8,66 & 7,94 \\
\hline 85 & 33,20 & 2,49 & 13,67 & 5,49 \\
\hline 86 & 19,40 & 1,26 & 9,49 & 7,56 \\
\hline
\end{tabular}

Les autres muscles occupent, selon les sexes, des positions très différentes dans les plans de projection des axes 1 et 2 et 1 et 3 . Chez les mâles, dans l'espace des 3 premiers axes, la moitié des muscles $(N=15)(T F, S T, G M, G P$, GR, SM, AD, VL, FS, TC, EL, PT, LE, PO, FP) sont regroupés au centre de la projection en une sorte de noyau central, qui est entouré d'un cortège périphérique de variables plus discriminantes (VM, OB, RF, MG, LG, GB, GA, PE, VI et $P L)$. Chez les femelles la situation est un peu différente et le regroupement au centre ne concerne qu'un plus faible nombre de muscles $(N=10)(V L, S T, G M$, $S M, A D, E L, L E, P O, F P$ et $P E$ ) et les variables GP, GR, VL, FS, TC, PT et PL sont un peu moins centrées chez les femelles que chez les mâles.

Dans les deux sexes les individus occupent largement l'espace de projection sur les trois axes. Pour la plupart, les groupes raciaux peuvent se distinguer les uns des autres. Par exemple les Suffolk se distinguent des Texel et des Solognot par des valeurs plus fortes du rapport VM/SO et les lle-de-France et les Rava occupant une position intermédiaire. Les Texel et les Solognot se distinguent par la valeur du rapport $\frac{\mathrm{QF}}{\mathrm{SO}}$, plus faible chez les premiers (fig. 3 et fig. 4).

b) Projection de l'ensemble des vingt quatre muscles. - L'élimination de l'analyse des 6 variables les plus discriminantes vise à mieux étudier les interrelations existant entre les autres variables et que la première analyse ne permettait pas de préciser suffisamment nettement.

La variation expliquée par les premiers axes est comparable dans les deux sexes et conduit aux mêmes conclusions que précédemment sur l'existence 
d'équilibres successifs entre variables pour structurer la population et expliquer la variation qu'on y rencontre (tabl. 4).

Les variables déterminantes des cinq premiers axes sont les mêmes dans les deux sexes, mais leur importance relative dans l'ordre de la hiérarchie n'est pas semblable. Les oppositions des variables dans l'espace déterminent des axes par-

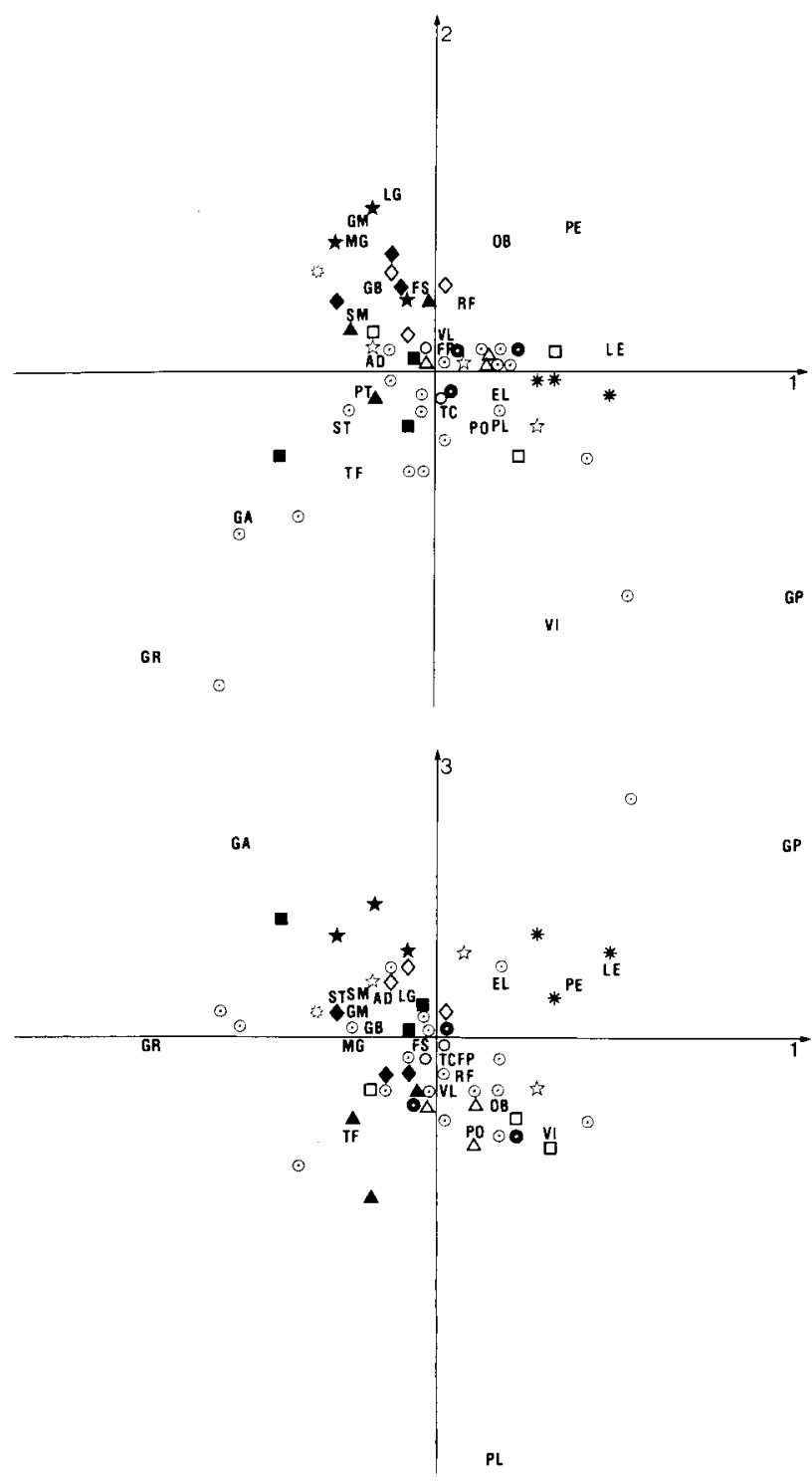

FIG. 5. - Plan de projection de l'ensemble des 24 muscles avec l'ensemble des animaux sur le premier et le deuxième axe (au-dessus) et le premier et le troisième axe (au-dessous) chez les mäles.

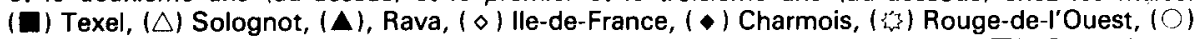
Vendéen, $(*)$ Suffolk, (is) Charolais, $(*)$ Romanov, (0) Tarasconnais, ( $\square$ ) Southdown, (Q) Agneau croisé. 
ticuliers de classement des animaux, spécialement chez les mâles, les axes GP/GR, GR/PE, GP/(LG, MG), PL/GA, PL/GP ; et chez les femelles, les axes $\mathrm{OB} / \mathrm{PL}, \mathrm{OB} / \mathrm{PT}, \mathrm{OB} / \mathrm{GA}, \mathrm{PT} / \mathrm{GA}, \mathrm{GP} / \mathrm{PL}$ (fig. 5 et fig. 6).

Dans les plans de projection définis par les axes 1, 2 et 1, 3 les différentes autres variables sont assez disséminées, ce qui traduit une grande variation dans

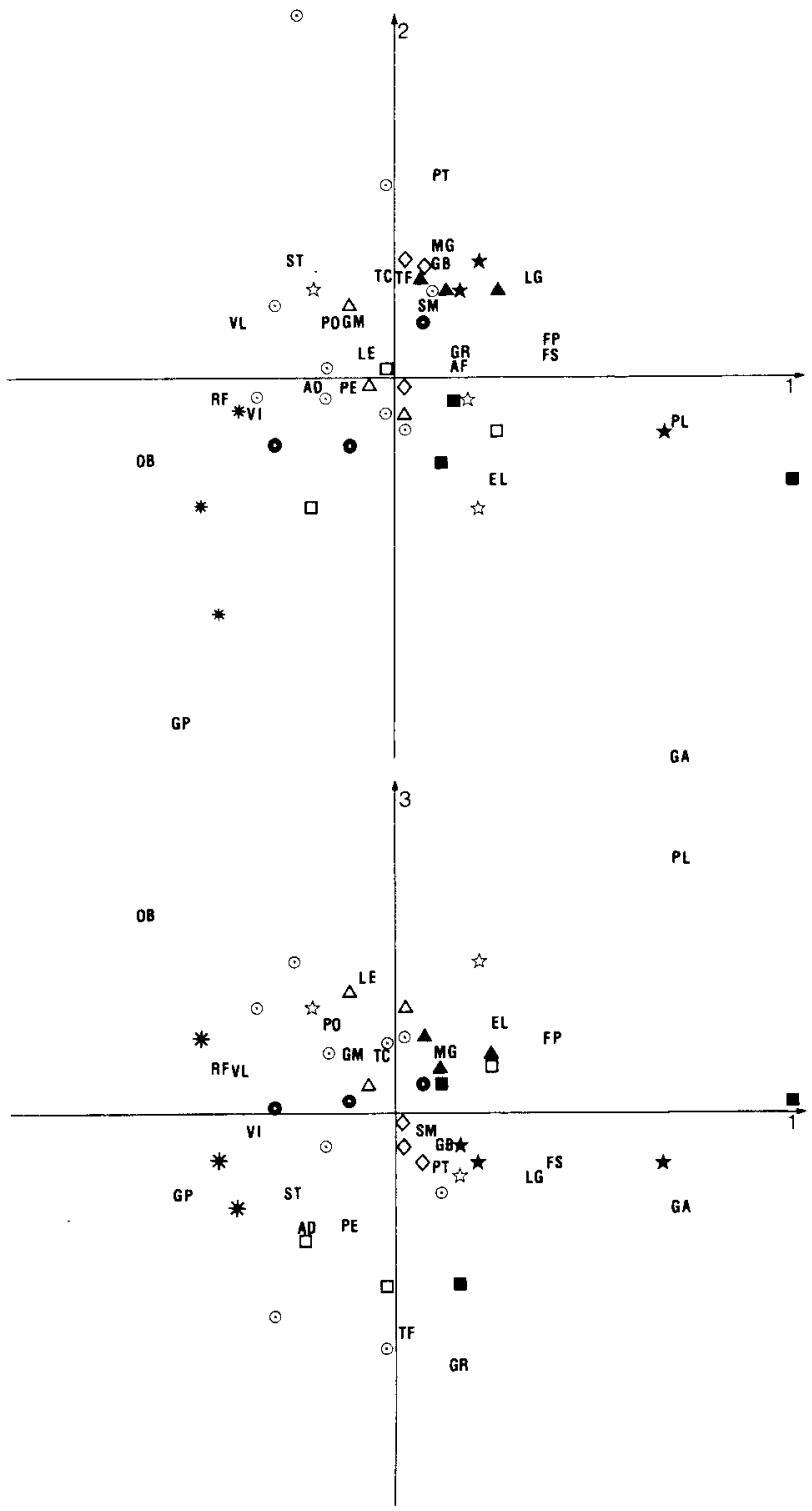

FIG. 6. - Plan de projection de l'ensemble des 24 muscles avec l'ensemble des animaux sur le premier et le deuxième axe (au-dessus) et le premier et le troisième axe (au-dessous) chez les femelles.

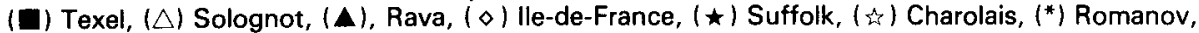
(•) Tarasconnais, ( $\square$ ) Southdown, $(\otimes)$ Agneau croisé. 
le rôle qu'elles peuvent jouer dans la discrimination des individus, donc dans l'explication de la variation existant dans la population. Dans les deux sexes on trouve relativement groupés au centre GB, SM, AD, TC et $P O$ et en plus chez les mâles, VL, RF, PT, FS et FP et chez les femelles ST, GM, PE, MG et LE.

Dans le plan des axes 1, 2 et 1, 3 les individus occupent largement l'espace de projection délimité par les variables les plus discriminantes. On remarque toutefois chez les mâles que rares sont les individus à' rapport GR/LG ou VI/LG ou $\mathrm{PL} / \mathrm{GA}$ et PL/GP élevés (fig. 5 et fig. 6).

Malgré une variabilité intragroupe quelquefois importante pour certains, il est possible de séparer précisément la plupart des groupes raciaux les uns des autres.

c) Projection des onze muscles les plus lourds. - On observe encore une évolution progressive de l'explication de l'inertie de la population par les différents axes successifs mais la part des premières variables explicatives de l'axe 1 et de l'axe 2 est ici plus importante, spécialement chez les mâles. L'essentiel de la variation est donc expliqué par quelques muscles seulement qui ne sont pas exactement les mêmes chez les mâles et les femelles (tabl. 4).

Dans l'espace de projection des trois premiers axes la disposition des variables est relativement différente d'un sexe à l'autre. On trouve cependant dans les deux sexes une opposition triangulaire entre VI, TF et LG $(V)$ et $L G$ s'opposant sur l'axe 1 et $\mathrm{VI}$ et LG s'opposant à TF sur l'axe 2), ce qui définit des axes particuliers de classement des individus (VI/LG, VI/TF et LG/TF). L'opposition entre LG et TF est toutefois moins marquée chez les femelles (fig. 7 et fig. 8).

Les variables $A D$ et $F P$, peu discriminantes chez les mâles, dans le plan des axes 1 et 2, prennent chez les femelles une grande importance ce qui conduit à considérer les axes VI/AD ou FP/AD comme structurant la population des femelles. Sur l'axe 3 l'opposition de RF à AD et ST est plus nette chez les mâles que chez les femelles où l'opposition RF/TF est, par contre, nettement marquée.

A partir du quatrième axe la situation est nettement différente chez les mâles et les femelles tant en ce qui concerne la nature des variables discriminantes que leur importance. On note également que les muscles les plus lourds (GB, SM, GM) n'interviennent que très secondairement dans la structuration de la population et par conséquent leur variation relative est de très faible importance.

Dans les deux sexes les individus occupent l'espace de projection sur les trois axes et il est possible de séparer les groupes raciaux les uns des autres (fig. 7 et fig. 8).

\section{Discussion générale.}

L'objectif de ce travail est une contribution à l'étude de la variabilité de la distribution musculaire dans le membre postérieur de l'espèce ovine. Cela nécessite qu'on considère un échantillon d'animaux représentatif de la variabilité existant dans l'espèce en ce qui concerne les caractères morphologiques, au plan notamment du format et de la conformation. Cette variabilité a été observée entre animaux de différentes races (et entre produits de croisement) dont les tailles étaient 

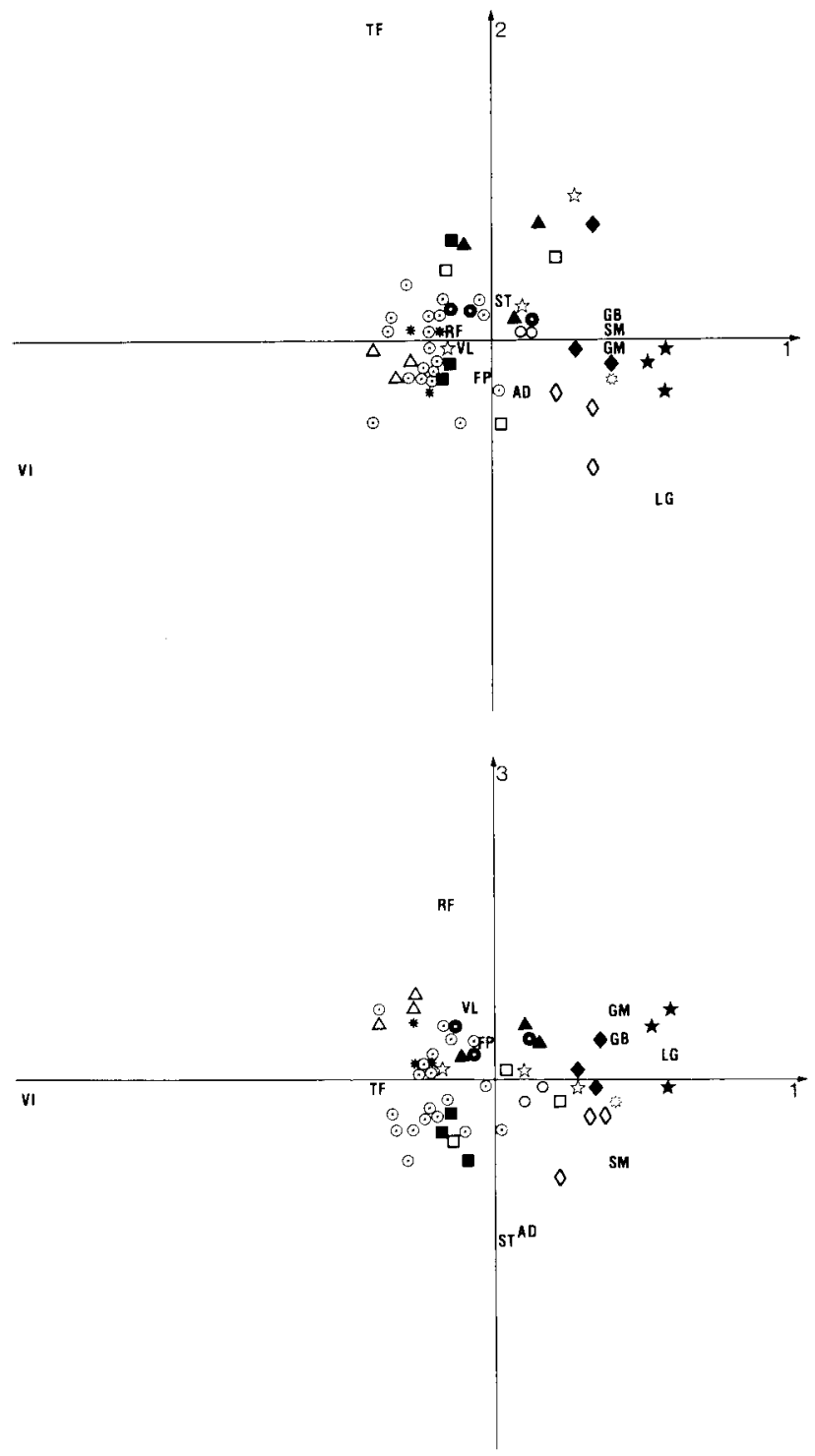

FIG. 7. - Plan de projection de l'ensemble des 11 muscles les plus lourds avec l'ensemble des animaux sur le premier et le deuxieme axe (au-dessus) et le premier et le troisieme axe (au-dessous)

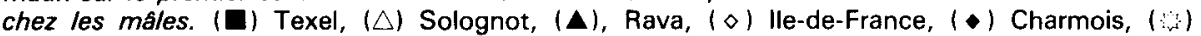
Rouge-de-l'Ouest, (O) Vendéen, $(\star)$ Suffolk, ( 4$)$ Charolais, (*) Romanov, ( $\bullet$ ) Tarasconnais, $(\square)$ Southdown, $(\otimes)$ Agneau croisé.

différentes et dont par la suite les masses étaient différentes puisque, pour comparer entre eux des animaux comparables au plan de la croissance musculaire, on a retenu de considérer, dans l'échantillon, des animaux d'état relatif de développement corporel voisin, en choisissant dans chaque sexe des sujets correspondant à 

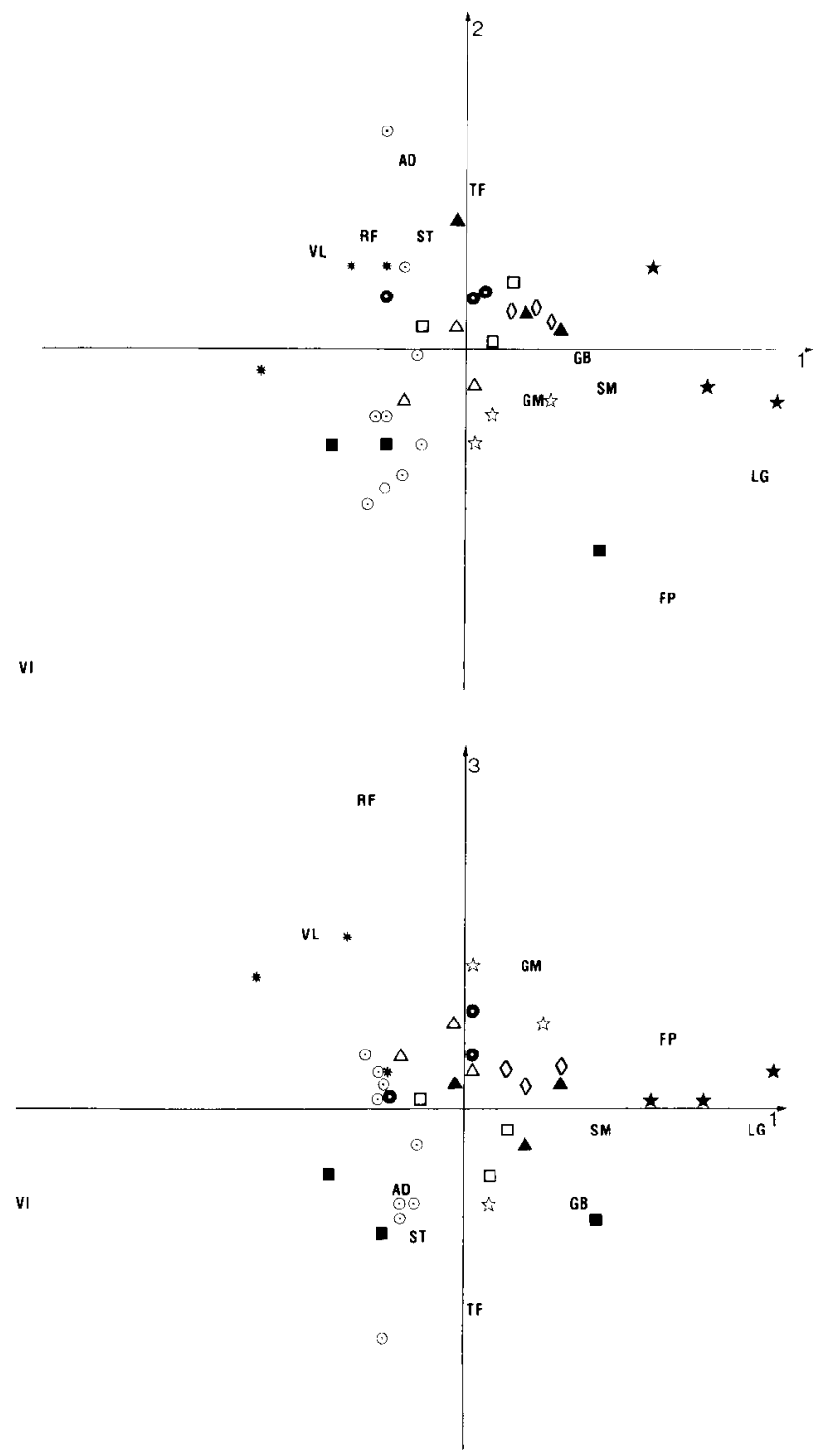

FIG. 8. - Plan de projection de l'ensemble des 11 muscles les plus lourds avec l'ensemble des animaux sur le premier et le deuxième axe (au-dessus) et le premier et /e troisième axe (au-dessous)

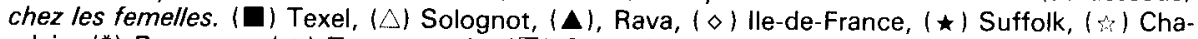
rolais, $(*)$ Romanov, $(\bullet)$ Tarasconnais, $(\square)$ Southdown, $(\otimes)$ Agneau croisé.

la catégorie des agneaux de boucherie du marché français (qui regroupe des animaux de 3 à 6 mois d'âge dont le poids d'abattage est de l'ordre de 40 pour 100 du poids adulte).

Cette condition implique une variabilité de base inévitable, qui se traduit, 
dans l'ensemble, par la variation enregistrée pour le poids total des muscles du membre.

La variabilité enregistrée dans la masse des muscles individuels s'explique en partie par un effet " taille » des individus. On note cependant que la variabilité n'est pas uniforme et qu'elle est dans l'ensemble très forte dans le cas des muscles de petite taille et plus faible dans les muscles les plus importants. On retrouve là une situation déjà observée par Van der Stelt et Klijn (1975) chez la grenouille et qui concorde bien, selon ces auteurs, avec le principe de Schrödinger (1945) selon lequel les organes possédant un plus grand nombre d'éléments - ici de fibres musculaires - ont, en général, des structures complexes plus stables. On peut ajouter que la plus grande variabilité de certains muscles pourrait s'expliquer aussi par les difficultés de leur reconnaissance anatomique et de leur séparation au cours de la dissection. Il est difficile, par exemple, de séparer $M$. vastus medialis du $M$. vastus intermedius ainsi que $M$. obturatorius externus du $M$. obturatorius internus. Cette hypothèse est, en fait, invérifiable expérimentalement, puisqu'on ne peut répéter l'opération de dissection sur le même matériel et donc pas déterminer l'importance de l'erreur expérimentale. A l'issue des résultats obtenus, la réévaluation des techniques de dissection utilisée à laquelle nous nous sommes livrés, de même que la prise en considération de la constance de l'ordre de prélèvement des muscles, nous amènent à conclure que la variabilité constatée ne nous semble pas imputable à des erreurs de dissection mais qu'elle doit avoir une origine propre, intrinsèque, caractéristique de chacun des muscles.

La variabilité de la masse de nombreux muscles est largement indépendante de celle des autres et cette indépendance conduit à déterminer, au sein de la musculature, entre muscles particulièrement variables, des rapports qui apparaissent être, comme le montre l'analyse des données centrées, des caractères très importants pour expliquer la variation constatée entre individus dans la distribution musculaire. Ces rapports ont ainsi valeur de rapports d'organisation des masses musculaires entre elles et permettent de classer les individus.

Sur l'ensemble des rapports mis en évidence par les axes successifs de projection, il est possible de positionner précisément les animaux dans l'espace multidimensionnel. Les analyses successives ont permis de définir ainsi une série de rapports impliquant finalement un nombre limité de muscles et ne concernant pratiquement pas les muscles les plus lourds.

La variation relative des masses musculaires décelée par ces rapports est importante et conduit à conclure que la distribution musculaire est, pour certains muscles, notablement variable. Ces résultats ne permettent donc pas pour le membre postérieur de considérer positivement l'hypothèse de l'existence d'une homéostasie musculaire traduite par une distribution de la musculature semblable chez tous les animaux comme cela a été suggéré par certains auteurs, chez les ovins et chez les bovins au niveau de l'ensemble de la musculature (Butterfield 1965 ; Charles et Johnson, 1976 ; Mukhoty et Berg, 1973 ; Bergström, 1978 ; Jury, Fourie et Kirton, 1977 ; Butterfield et al., 1983 ; Jackson et Mansour, 1974 ; Kauffman et al., 1973). Les résultats observés ici correspondent, au contraire, à l'existence d'une variabilité de la distribution musculaire, en accord avec les 
observations faites chez les ovins ou les bovins [Hammond (1932); Seebeck (1973) ; Martin, Walters et Whiteman (1966); Truscott et al. (1976)].

La séparation des groupes d'animaux selon leur type dans les analyses des données centrées montre que la distribution musculaire est caractéristique de chaque type génétique. On remarque d'autre part que dans les projections des différentes analyses on trouve côte à côte des groupes d'agneaux de niveaux de conformation différents, ce qui conduit à penser que la distribution musculaire n'est pas affectée par la conformation.

L'origine de ces différences n'est pas facile à établir en raison de la grande diversité des fonctions et des localisations au sein du membre postérieur des principaux muscles discriminants. Une origine unique des différences pour tous les muscles concernés ne semble pas pouvoir être avancée, pour l'instant. Toutefois, les différences constatées par ailleurs dans les caractères des supports osseux des individus (masse relative, dimensions du bassin, du fémur, du tibia) sont à rapprocher des différences dans le développement relatif de certains des muscles entre eux. Ce point est en cours d'étude dans notre laboratoire (Anous, 1986b).

La comparaison des résultats obtenus dans les deux sexes montre une certaine ressemblance entre les mâles et les femelles. Mais il est cependant possible de conclure à un effet du sexe sur la distribution musculaire.

L'ampleur de la variation de la distribution musculaire mise en évidence par cette étude traduit, d'après notre hypothèse et le choix de notre matériel animal, l'effet sur ce caractère de la variabilité observée dans l'espèce au plan morphoanatomique. A cet égard, la portée de notre travail doit être appréciée en remarquant que le choix des animaux n'a pu être effectué, pour des raisons matérielles, qu'à l'intérieur de la population ovine française. D'autre part, malgré le soin apporté pour comparer des agneaux d'état semblable de développement corporel, il n'est pas possible d'affirmer absolument que les différences constatées entre individus dans la part relative des muscles ne peuvent pas, pour une part, être imputés à des différences de maturité de développement musculaire. Ce point ne pourra être élucidé qu'en comparant, de manière systématique dans les divers types génétiques, la variation de la distribution musculaire au cours de la croissance. Ce point est en cours d'investigation dans notre laboratoire pour deux types génétiques.

\section{Conclusion.}

L'étude a montré que les variables déterminantes des premiers axes sont les mêms dans les deux sexes, mais leur importance relative dans l'ordre de la hiérarchie n'est pas semblable. Dans les deux sexes, la plupart des groupes raciaux peuvent se distinguer les uns des autres sur la base de différents rapports entre les variables les plus discriminantes. Au total, il semble donc qu'on ne puisse pas parler d'une distribution uniforme de la musculature du membre postérieur des ovins et qu'il n'existe pas, au niveau des muscles individuels, une homéostasie stricte de la composition musculaire. 
Remerciements. - Je remercie M. Dumont pour les critiques et les suggestions faites dans la préparation du manuscrit, M. Bousset pour l'aide apportée dans le traitement informatique, Melle Martin pour la réalisation des illustrations et Mme Besnard pour la dactylographie du texte.

Je remercie également pour l'approvisionnement en animaux nécessaires à cette étude $M$. Hondermark et M. Lecloux (de I'ITOVIC).

\section{Références}

ABDALLAH O. Y., MAHALLAWI M., LATIF M.G.A., 1982. Anatomical and joint dissection studies of tissue weight distribution in Buffalo bulls and cows : Part I. - Distribution of muscle and bone. Meat Sci, 7, 117-129.

ANOUS M. R. I., 1980 Some studies on growth patterns and meat production in Egyptian goats and sheep. M. Sc. Thesis, Dept. anim. Prod., Faculty of Agriculture, Ain-Shams University, Cairo, Egypt.

ANOUS M. R., 1986a. Interrelations entre principaux composants anatomiques, conformation et longueur des os du membre postérieur des ovins. Ann. Zootech. (soumis pour publication).

ANOUS M. R., 1986b. Variabilité des caractéristiques morphologiques des supports osseux du membre postérieur de l'agneau (travaux en cours).

BARONE R., 1980. Anatomie comparée des mammifères domestiques. Tome 2. Arthrologie et Myologie. Vigot, Paris.

BÉNÉVENT M., 1968. Guide pour la dissection et l'identification des principaux muscles de la carcasse chez le mouton. Ann. Biol. anim. Bioch. Biophys., 8, 147-193.

BERGSTRÖM P. L., 1978. Sources of variation in muscle weight distribution. Curr. Topics Vet. Med., Vol. 2, 91-131.

BOCCARD R., DUMONT B. L., 1960. Etude de la production de la viande chez les ovins. II. - Variation de l'importance relative des différentes régions corporelles de l'agneau de boucherie. Ann. Zootech., 9, 355-363.

BOCCARD R., DUMONT B. L., 1973. Etude de la production de la viande chez les ovins. IX. - Variation de l'organisation de la musculature de l'agneau en fonction de la vitesse de croissance. Ann. Zootech., 22, 423-431.

BOCCARD R., DUMONT B. L., LE GUELTE P., ARNOUX J., 1961. Etude de la production de la viande chez les ovins. IV. - Relation entre la forme et la composition du membre postérieur. Ann. Zootech., 10, 155-160.

BUTLER-HOGG B. W., WHELEHAN O. P., 1984. Fat partitioning and muscle distribution in Texel and Scottish Blackface rams. 35th Meet. EAPP, 6th-9th August 1984. The Hague, Holland.

BUTTERFIELD R.M., 1965. Practical implications of anatomical research in beef cattle. Proc. N.Z. Soc. anim. Prod., 25, 152-163.

BUTTERFIELD R. M., JOHNSON E. R., 1968. The effect of growth rate of muscle in cattle on conformation as influenced by muscle weight distribution, 212-223. In Growth and development of mammals. Butterworths, London.

BUTTERFIELD R. M., REDDACLIF K. J., THOMPSON J. M., ZAMORA J., WILLIAMS J., 1984. Changes in body composition relative to weight and maturity of Australian Dorset Horn rams and wethers. 2. Individual muscles and muscle groups. Anim. Prod., 39, 259-267.

BUTTERFIELD R. M., ZAMORA J., JAMES A. M., THOMPSON J. M., WILLIAMS J., 1983. Changes in body composition relative to weight and maturity in large and small strains of Australian Merino rams. 2. Individual muscles and muscle groups. Anim. prod., 36, 165-174.

CHARLES D. D., JOHNSON E. R., 1972. Carcass composition of the water buffalo (Buba/us bubalis). Aust. J. agric. Res., 23, 905-911.

CHARLES D. D., JOHNSON E. R., 1976. Muscle weight distribution in four breeds of cattle with reference to individual muscles, anatomical groups and wholesale cuts. J. agric. Sci, Camb., 86, 435-442.

COLOMER-ROCHER F., BASS J. J., JOHNSON D. L., 1980 Beef carcass conformation and some relationships with carcass composition and muscle dimensions. J. agric. Sci., Camb., 94, 697-708. 
DAVIES A. S., 1974. A comparison of tissue development in Pietrain and Large White pigs from birth to $64 \mathrm{~kg}$ live weight. Anim. Prod., 19, 377-387.

DUMONT B. L., 1983. Relations entre des caractères à signification de développement musculaire dans la carcasse de porc. Journées Rech. Porcine en France, 15, 233-238.

DUMONT B. L., BOULLEAU T., 1984. Note sur l'influence du type génétique sur la composition du membre postérieur du porc femelle. Comparaison Landrace Belge, Landrace Français, Piétrain. Journées Rech. Porcine en France, 16, 95-102.

DUMONT B. L., LE GUELTE P., ARNOUX J., 1961. Etude biométrique des bovins de boucherie. I. - Variabilité de la composition anatomique de la carcasse des bovins Charolais. Ann. Zootech., 10, 149-154.

DUMONT B. L., ROY G., 1975. Etude comparative des caractères du membre postérieur chez les Landrace Belge, Landrace Français et Pietrain. Journées Rech. Porcine en France, 7, 195202.

DUMONT B. L., SCHMITT O., ROY G., 1969. Développement musculaire comparé de porcs Piétrain et Large-White. Rec. Méd. vét., Ecole Alfort, 145, 937-947.

FOURIE P. D., 1965. Growth and development of sheep, with special reference to new Zealand breeds. D. Sc. (Agric.) Thesis, University of Pretoria.

GAILI E. S. E., 1976. Body composition of male Sudan desert goats. World Rev. anim. Prod., Vol. XII, N ${ }^{\circ} 4,83-87$.

GAILI E. S. E., ALI A. E., 1985. Meat from Sudan desert sheep and goats : Part 1 - Carcass yield, offals and distribution of carcass tissues. Meat Sci., 13, 217-227.

GETTY R., 1975. Sisson and Grossman's, The anatomy of the domestic anima/s. 5th ed., W. B. Saunders Co. Philadelphia, London, Toronto.

GOENAGA P. R., CARDEN A. E., 1979. A comparison of tissue weight distribution in Landrace, Hampshire and Duroc Jersey pigs. J. agric. Sci., Camb., 93, 271-280.

HAMMOND J., 1932. Growth and the development of mutton qualities in the sheep. Oliver and Boyd, Edinburgh.

JACKSON T. H., MANSOUR Y. A., 1974. Differences between groups of lambs carcasses chosen for good and poor conformation. Anim. Prod., 19, 93-105.

JURY K. E., FOURIE P. D., KIRTON A. H., 1977. Growth and Development of Sheep. IV. Growth of the Musculature. N. Z. J. agric. Res., 20, 1115-1121.

KAUFFMAN R. G., GRUMMER R. H., SMITH R. E., LONG R. A., SHOOK G., 1973. Does liveanimal and carcass shape influence gross composition ? J. anim. Sci, $37(5), 1112-1119$.

KEMPSTER A. J., CUTHBERTSON A., SMITH R. J., 1976. Variation in lean distribution among steer carcasses of different breeds and crosses. J. agric. Sci., Camb., 87, 533-542.

KLIJN H. B., 1976. An analysis of muscle weight variations in the wing and leg of Sturnus vulgaris. Anat. Embryol., 149, 259-287.

LEFEBVRE J., 1976. Introduction aux analyses statistiques multidimensionnelles. $219 \mathrm{pp}$. Masson, Paris.

MARTIN E. L., WALTERS L. E., WHITEMAN J. V., 1966. Association of beef carcass conformation with thick and thin muscle yields. $J$, anim. Sci, 25, 682-687.

MAY N. D. S., 1964. Anatomy of the sheep. 2nd ed., Univ. of Queensland Press, Brisbane, Australia,

MUKHOTY H., BERG R. T., 1973. Influence of breed and sexe on muscle weight distribution of cattle. J. agric. Sci., Camb., 81, 317-326.

OWEN J. E., ARIAS CERECERES M. T. A., GARCIA MACIAS J. A., NUÑEZ GONZALEZ F. A., 1983. Studies on the Criollo goat of Northern Mexico : Part 1 - The effects of body weight on body components and carcass development. Meat Sci, 9, 191-204.

OWEN J. E., NORMAN G. A., PHILBROOKS C. A., JONES N. S. D., 1978. Studies on the meat production characteristics of Botswana goats and sheep. Part III : Carcass tissue composition and distribution, Meat Sci., 2, 59-74.

PÁLSSON H., 1940. Meat qualities in the sheep with special reference to Scottish breeds and crosses. Part III : Comparative development of selected individuals of different breeds and crosses as lambs and hoggets. J. agric. Sci., Camb., 30, 1-64.

RICHMOND R. J., BERG R. T., 1971. Muscle growth and distribution in suine as influenced by liveweight, breed, sex and ration. Can. J. anim. Sci., 51, 41-49. 
ROUVIER R., RICARD F. H., 1967. Etude de la composition anatomique du poulet. II. - variabilité de la répartition de la viande et de l'os chez des coquelets "Bresse-Pile ». Ann. Zootech., 16. 357-374.

SCHRÖDINGER E., 1945. What is life ? The physical aspect of the living cell. Cambridge University Press, London.

SEEBECK R. M., 1973. The effect of body-weight loss on the composition of Brahman cross and Africander cross steers. II. - Dissected components of the dressed carcass. J. agric. Sci, Camb., 80, 411-423.

STELT A. Vand der, KLIJN H. B., 1975. An analysis of muscle weight variations in the hindlimbs of Rana esculenta. Anat. Embryol., 147, 225-241.

TAYLOR ST C. S., MASON M. A., McCLELLAND T. H., 1980. Breed and sex differences in muscle distribution in equally mature sheep. Anim. Prod., 30. 125-133.

TEYSSIER J., PRUD'HON M., 1982. Evaluation des différences génétiques dans l'importance et la répartition du tissu musculaire, entre 25 et 150 jours, sur des agneaux Romanov Berrichon $\times$ Romanov, Texel $\times$ Romanov, Lacaune $\times$ Romanov, Charmois $\times$ Romanov. Ann. Génét. Sél. anim., 14, 353-368.

THORGEIRSSON S., 1981. Growth and development of Scottish Blackface and /celandic sheep. $\mathrm{Ph}$. D. Thesis, School of Agriculture, University of Edinburgh.

TRUSCOTT T. G., LANG C. P., TULLOH N. M., 1976. A comparison of body composition and tissue distribution of Friesian and Angus steers. J. agric. Sci., Camb., 87, 1-14.

WILSON P. N., 1958. The effect of plane of nutrition on the growth and development of the East African Dwarf goat. Part II. Age changes in the carcass composition of female kids. J. agric. Sci., Camb., 51, 4-22. 